observed among BRCA2 heterozygous patients (p.Q3299X: n = 2; c.5345dupA: $\mathrm{n}=1$; c.7408_7409delTT: $\mathrm{n}=1$ ). Given the high frequency of mutations in BRCA1 gene, we added to the study 44 consecutive patients with triple-negative breast cancer. This effort relied on the fact, that BRCA1 is specifically associated with the triple-negative phenotype of breast cancer disease; 3 (7\%) additional BRCA1 mutation carriers (c.3627_3628delAG: $\mathrm{n}=2$; c.1338_1339delAG: $\mathrm{n}$ $=1$ ) were revealed. All patients with the BRCA1 c.3627_3628delAG pathogenic variant also carried linked c.1067 $\overline{\mathrm{G}}>\mathrm{A}$ (p.Q356R) polymorphic substitution; therefore, BRCA1 c.3627_3628delAG is indeed a founder allele, but not a mutational hot spot. In addition to BRCA1/2, one HGSOC patient carried ATM truncating variant (p.Q1171X). There were no instances of PALB2 or TP53 germline alterations.

Conclusion* This is a small-scale study, which resulted in convincing demonstration of a strong founder effect in Chechen women with hereditary breast-ovarian cancer. Genetic testing of non-selected HGSOC patients allows highly efficient analysis of ethnicity-specific spectrum of BRCA1/2 mutations.

\section{GYNECOLOGIC MALIGNANCIES IN THE ERA OF PRECISION MEDICINE}

M Mantiero*, M Ducceschi, M Bini, S Lopez, M Duca, S Damian, A Ditto, F Martinelli, U Leone Roberti Maggiore, G Bogani, M Signorelli, F Bertolina, V Chiappa, B Paolini, L Agnelli, F Raspagliesi. Fondazione IRCCS Istituto Nazionale dei Tumori di Milano, Milan, Italy

\subsection{6/ijgc-2021-ESG0.589}

Introduction/Background* Personalized medicine is replacing the classical one-size-fits-all traditional oncology approaches tailoring the most appropriate therapy for each patient. Molecular and genomic profiling diagnostic tools are implementing patients' journey.

Methodology This is a single centre prospective study performed from January 2020 and April 2021 at Fondazione IRCCS Istituto Nazionale dei Tumori di Milano (Italy). All consecutive, heavily pretreated patients, for whom effective conventional treatments were not available, were enrolled in this study and underwent molecular and genomic profiling via Foundation One CDx test.

Result(s)* Overall, 63 heavily pretreated patients had Foundation One CDx test. We identified 10 patients (16\%) with mutation or genetic signatures candidate to use personalized therapy (table 1). Out of 10 patients, 4 are patients affected by cervical, 4 by endometrial and the remnant are affected by ovarian carcinoma. Actually 1 patient is receiving immunotherapy with atezolizumab plus anti-ICOS and 1 is undergoing evaluation in order to start same drugs; 2 patients with ovarian cancer had BRAF/V600E mutations and are ongoing on treatment with trametinib +/- dabrafenib; 2 patients with cervical cancer had PI3KCA mutations and are treating with alpelisib. Furthermore, in 4 patients an actionable mutation was found but standard chemotherapeutic treatment is still ongoing (Table 2).

Immunotherapy and target therapy are administered into the clinical trial or thank to compassionate use.

Conclusion* Molecular and genomic profiling of gynecological malignancies is not clinical practice. We demonstrated that in
Abstract 319 Table 1 Global patients

\begin{tabular}{lc}
\hline Patients total N (\%) & $63(100)$ \\
Age median (range) & $59(29-78)$ \\
Primary tumor & $17(27)$ \\
Ovarian carcinoma & $5(8)$ \\
Endometrial carcinoma & $33(52)$ \\
Cervical carcinoma & $4(6)$ \\
Uterine sarcoma & $1(2) 2(3)$ \\
Vaginal carcinoma & \\
Bartholin's gland adenocarcinoma & \\
Target therapy & $10(16)$ \\
\hline
\end{tabular}

\begin{tabular}{|c|c|c|c|c|}
\hline Patient & Age & Pathology & Mutation & Target therapy \\
\hline 1 & 77 & $\begin{array}{l}\text { Clear cell endometrial } \\
\text { carcinoma }\end{array}$ & $\begin{array}{l}\text { mut. L755S } \\
\text { ERBB2 }\end{array}$ & Evaluable for Afatinib \\
\hline 2 & 67 & $\begin{array}{l}\text { High grade serous } \\
\text { ovarian carcinoma }\end{array}$ & Mut. V600E BRAF & $\begin{array}{l}\text { Ongoing Dabrafenib + } \\
\text { trametinib }\end{array}$ \\
\hline 3 & 54 & $\begin{array}{l}\text { Squamous cervical } \\
\text { carcinoma }\end{array}$ & $\mathrm{HPV}$ at ISH & $\begin{array}{l}\text { Ongoing Atezolizumab + } \\
\text { anti ICOS }\end{array}$ \\
\hline 4 & 52 & $\begin{array}{l}\text { Endometrial } \\
\text { carcinosarcoma }\end{array}$ & MSI & $\begin{array}{l}\text { Evaluable for } \\
\text { Atezolizumab }\end{array}$ \\
\hline 5 & 71 & Endometrial carcinoma & $\begin{array}{l}\text { Mut R88Q } \\
\text { PI3KCA }\end{array}$ & $\begin{array}{l}\text { Evaluable for Everolimus } \\
+ \text { exemestane }\end{array}$ \\
\hline 6 & 30 & $\begin{array}{l}\text { Mucinous cervical } \\
\text { adenocarcinoma }\end{array}$ & $\begin{array}{l}\text { splice site (134 } \\
+1 \mathrm{G}>\mathrm{T}) \text { PTEN }\end{array}$ & Evaluable for Everolimus \\
\hline 7 & 60 & $\begin{array}{l}\text { Low grade serous } \\
\text { ovarian carcinoma }\end{array}$ & Mut. V600E BRAF & Ongoing Trametinib \\
\hline 8 & 41 & $\begin{array}{l}\text { Squamous cervical } \\
\text { carcinoma }\end{array}$ & $\begin{array}{l}\text { Mut p.E545K } \\
\text { PI3KCA }\end{array}$ & Ongoing Alpelisib \\
\hline 9 & 71 & $\begin{array}{l}\text { Endometrioid } \\
\text { Endometrial carcinoma }\end{array}$ & $\begin{array}{l}\text { Mut. Q546P } \\
\text { PI3KCA }\end{array}$ & Evaluable for Alpelisib \\
\hline 10 & 39 & $\begin{array}{l}\text { Neuroendocrine cervical } \\
\text { cancer }\end{array}$ & $\begin{array}{l}\text { Mut p.E545K } \\
\text { PI3KCA }\end{array}$ & Ongoing Alpelisib \\
\hline
\end{tabular}

this population identified alterations, by genetic driver, could help to find a new therapeutically opportunity. This allows to identify predictive biomarkers for target therapies in order to offer new therapeutic prospective for our gynecologic patients.

\section{CHARACTERISATION OF INTRA-TUMOURAL HETEROGENEITY IN HIGH GRADE SEROUS OVARIAN CANCER}

${ }^{1} \mathrm{P}$ Cunnea*, ${ }^{1} \mathrm{E}$ Curry, ${ }^{2} \mathrm{E}$ Christie, ${ }^{1} \mathrm{~K}$ Nixon, ${ }^{1} \mathrm{CH}$ Kwok, ${ }^{1} \mathrm{~J}$ Ploski, ${ }^{2} \mathrm{D}$ Bowtell, ${ }^{1} \mathrm{C}$ Fotopoulou. ${ }^{1}$ Imperial College London, Hammersmith Campus, London, UK; ${ }^{2}$ Peter MacCallum Cancer Centre, Melbourne, Australia

\subsection{6/ijgc-2021-ESG0.590}

Introduction/Background* High-grade serous ovarian cancer (HGSOC) is typified by extensive genomic instability and intra-tumoural heterogeneity (ITH). The majority of patients relapse and eventually acquire resistance to platinum-based chemotherapy. Diverse mechanisms leading to platinum 
resistance and a lack of predictive biomarkers means that matching the best treatment options to patients is difficult. This study aims to characterise the extent of spatial and temporal ITH in advanced stage HGSOC at presentation and relapse.

Methodology Patients $(n=49)$ having maximal effort upfrontdebulking surgery for advanced HGSOC underwent a tumour mapping of their tumour dissemination patterns. Tumour biopsies were collected (range 4-15, median 9), when relapsed patients also had paired biopsies collected for genomic and phenotypic analysis. DNA was extracted from tumours (5 per patient, $n=49$ patients plus relapse samples) and Illumina Human OmniExpress genotyping performed. Allele-specific copy number $(\mathrm{CN})$ was quantified using ASCAT. Genomic heterogeneity was quantified as the estimated number of $\mathrm{CN}$ aberration events distinct between each pair of tumour deposits. Clonal diversity within a patient's deposits was calculated using the difference between within-patient and betweenpatient heterogeneity. Ki67 proliferation index was assessed from tumour sections collected prior to DNA extraction.

Result(s)* Extensive genomic variations in patterns of evolution for different patients' tumours was observed, including the relationship between matched primary tumours and relapsed disease. Widespread variations in CCNE1, MYC and PTEN CN were observed across multiple disseminated tumours in the same patients, and higher CCNE1 correlated with poor patient outcome $(p=0.038)$. Extensive heterogeneity in Ki67 proliferation index was observed across the cohort, $77 \%$ of patients had tumour scores covering low, moderate and/or high Ki67 scoring categories.

Conclusion* Broad ITH was observed at the genomic level across this cohort. Extensive $\mathrm{CN}$ variations in genes such as CCNE1, across multiple disseminated samples within patients, and widespread variations in proliferative index between multi-site tumours, indicates that a single tumour biopsy does not accurately depict disseminated HGSOC biology, and therefore should not be used for as a basis for prediction of patient prognosis or outcomes.

\section{RADIOMICS AND MOLECULAR CLASSIFICATION IN ENDOMETRIAL CANCER (THE ROME STUDY): A STEP FORWARD TO A SIMPLIFIED PRECISION MEDICINE}

${ }^{1} \mathrm{G}$ Bogani*, ${ }^{2} \mathrm{C}$ Castiglioni, ${ }^{1} \mathrm{~V}$ Chiappa, ${ }^{1} \mathrm{~F}$ Raspagliesi*. ' Istituto Nazionale dei Tumori | Fondazione IRCCS, Milano, Italy; ${ }^{2}$ Building U6 - University of Milano-Bicocca, Milano, Italy

\subsection{6/ijgc-2021-ESGO.591}

Introduction/Background* Molecular/genomic profiling is the most accurate method to assess prognosis of endometrial cancer patients. Similarly, the adoption of radiomics showed important results for screening, diagnosis and prognosis, across various radiological systems and oncologic specialties. Here, we aim to correlate radiomic features obtained from ultrasound images with the molecular/genomic profiling to identify new hallmarks for stratification of endometrial cancer patients into different classes of risk.

Methodology

This prospective single-arm observational study Patients with newly diagnosed endometrial cancer will have ultrasonographic evaluation and radiomic analysis of the ultrasonographic images. Then patients will have surgery followed by molecular/genomic evaluation. We will correlate radiomic features with molecular/genomic profiling to classify prognosis. Major Inclusion/Exclusion Criteria : Consecutive patients (aged 18 years or more) with newly diagnosed endometrial cancer. Patients should have preoperative ultrasonographic evaluation followed by surgery.

Result(s)* The central hypothesis is that combining radiomic features with molecular features might allow identifying various classes of risk for endometrial cancer, e.g. predicting unfavorable molecular/genomic profiling. The rationale for the proposed research is that once validated, radiomics applied to ultrasonographic images would be an effective, innovative and cheap method for tailor operative and postoperative treatment modality in endometrial cancer. Primary Endpoint : The main endpoints will: (i) to define the mechanism by which radiomic features predict the classification of endometrial cancer into various classes of risk e.g. predicting unfavorable molecular/ genomic profiling as defined by molecular classification; (ii) to determinate the scaled impact of radiomic features assessed on ultrasonographic images of endometrial tumors; and (iii) to assess the intraobserver and interobserver reproducibility of radiomic features on ultrasonographic images of endometrial tumors. Overall, 100 patients for study cohort and 40 for the validation cohort.

Conclusion* We expect that the radiomic analysis of ultrasonographic images by means of radiomic classifier of risks will provide comparable results to molecular/genomic. (Trial Registration: GR-2019-12370566 Bando per la Ricerca Finalizzata 2019, Ministero della 24 Salute, Repubblica Italiana)

\section{RAD51 RING TRIAL: A EUROPEAN INTERLABORATORY ANALYTICAL VALIDATION TO DETERMINE THE ROBUSTNESS OF RAD51 AS A BIOMARKER FOR HOMOLOGOUS RECOMBINATION}

${ }^{1} \mathrm{C}$ Kramer*, ${ }^{2} \mathrm{~A}$ Llop-Guevara, ${ }^{3} \mathrm{E}$ Yaniz-Galende, ${ }^{4} \mathrm{~B}$ Pellegrino, ${ }^{1} \mathrm{~N}$ Ter Haar, ${ }^{4} \mathrm{~N}$ Campanini, ${ }^{4} \mathrm{~A}$ Musolino, ${ }^{3} \mathrm{~A}$ Leary, ${ }^{5} \mathrm{M}$ Vreeswijk, ${ }^{2} \mathrm{~V}$ Serra, ${ }^{1} \mathrm{~T}$ Bosse. ${ }^{1}$ Leiden University Medical Center (LUMC), Pathology, Leiden, Netherlands; '2VHIO Vall d'Hebron Institut d'Oncologia, Experimental Therapeutics Group, Barcelona, Spain; ${ }^{3}$ Hospital Gustave Roussy, Oncological Medicine, Villejuif, France; ${ }^{4}$ University Hospital of Parma, Medical Oncology and Breast Unit, Medicine , Parma, Italy; ${ }^{5}$ Leiden University Medical Center (LUMC), Human Genetics, Leiden, Netherlands

\subsection{6/ijgc-2021-ESG0.592}

Introduction/Background* RAD51 protein has been proposed as a functional readout of homologous recombination (HR) status using formalin-fixed paraffin embedded (FFPE) tumour tissue blocks. Recently, few laboratories have assessed the performance of RAD51 as a predictive biomarker. However, the robustness of the test when applied in different laboratories has not been systematically investigated so far. In this study, we performed an interlaboratory $(n=4)$ analytical validation to determine the interobserver variability and the effect of (subtle) differences in the co-immunofluorescence (co-IF) protocol and microscope technicalities on RAD51 scores.

Methodology The RING trial cohort comprised of 12 highgrade serous ovarian cancer cases. On unstained serial sections of FFPE tumour tissue blocks, a co-IF staining with RAD51 and geminin was performed: (1) centrally in Vall d'Hebron Institute of Oncology and (2) locally in participating centers. The centrally stained slides were distributed among 